Revue d'histoire de l'Amérique française

Z7Q REVUE D.HISTOIRE DE L'AMÉRIQUE FRANÇAISE

\title{
Les archives notariales et l'histoire sociale de la Nouvelle-France
}

\section{Louis Lavallée}

Volume 28, numéro 3, décembre 1974

URI : https://id.erudit.org/iderudit/303369ar

DOI : https://doi.org/10.7202/303369ar

Aller au sommaire du numéro

Éditeur(s)

Institut d'histoire de l'Amérique française

ISSN

0035-2357 (imprimé)

1492-1383 (numérique)

Découvrir la revue

Citer cette note

Lavallée, L. (1974). Les archives notariales et l'histoire sociale de la

Nouvelle-France. Revue d'histoire de l'Amérique française, 28(3), 385-403.

https://doi.org/10.7202/303369ar d'utilisation que vous pouvez consulter en ligne.

https://apropos.erudit.org/fr/usagers/politique-dutilisation/ 


\title{
LES ARCHIVES NOTARIALES ET L'HISTOIRE SOCIALE DE LA NOUVELLE-FRANCE
}

\author{
LOUIS LAVALLÊE \\ Département d'histoire \\ Université de Montréal
}

Depuis une vingtaine d'années, l'intérêt tout particulier porté par les historiens français aux structures sociales, aux groupes sociaux, aux problèmes de stratification et de classification, à l'histoire sociale en général, assimilée le plus souvent à une forme d'histoire totale, a amené ceux-ci à rechercher un type de documentation archivistique qui, tout en se prêtant à cette histoire sérielle chère à Pierre Chaunu, pouvait leur fournir les matériaux nécessaires pour l'étude du social inscrit dans un cadre temporel de longue durée, vérité commune acceptée depuis la thèse célèbre que signait Fernand Braudel il y a vingtcinq ans. ${ }^{1}$ Les premiers écrits autour des années cinquante avaient pour tâche de préciser les buts à accomplir et devaient délimiter le genre d'archives qui étaient susceptibles de répondre adéquatement aux questions posées sur le groupe et les structures de la société.

A cet égard, la communication présentée par Ernest Labrousse au Congrès international des Sciences historiques à Rome en $1955^{2}$ a marqué une date importante et fut le point de départ de nombreux travaux qui allaient utiliser par la suite des documents d'archives jusque-là négligés le plus souvent par les historiens. En effet, Labrousse mettait en évidence et rappelait la richesse exceptionnelle des sources fiscales et des minutes notariales dans la quête de la stratification et de la définition des groupes sociaux. Subséquemment, de nombreux disciples et des écoles diverses se sont lancés avec enthousiasme dans le dépouillement systématique des archives fiscales ou notariales

${ }^{1}$ C'est en 1949 que paraît La Méditerranée et le monde méditerranéen à l'époque de Philippe II.

2 Ernest Labrousse, Voies nouvelles vers une histoire de la Bourgeoisie occidentale aux XVIIle et XIXe siècles (1700-1850) - X Congrès international des Sciences historiques (Rome, 1955), Relazioni, vol. IV, Storia Moderna: 365-396.

RHAF, vol. 28, no 3 (décembre 1974) 
et les premiers résultats ont vu le jour en 1961 quand Adeline Daumard et François Furet ont présenté cet essai d'histoire sociale intitulé: Structures et relations sociales à Paris au XVIII siècle. Depuis, de nombreux articles et monographies ont paru en France sous la direction d'Ernest Labrousse et de Roland Mousnier principalement, ce dernier s'appliquant particulièrement à critiquer les options trop essentiellement économiques de certains historiens. Un fait demeure, depuis quelques décennies, l'histoire sociale est en France devenue principalement tributaire de ces deux grandes catégories d'archives et l'expérience acquise par les usagers de cette documentation a donné lieu à une mise au point des méthodes d'utilisation et d'exploitation.

L'histoire sociale du Canada français et tout spécialement celle de la Nouvelle-France à laquelle nous voulons nous attacher de plus près sont également attentives aux recherches nouvelles susceptibles d'enrichir notre savoir sur les sociétés d'Ancien Régime. Notre but est simple. Nous voulons rendre compte de l'intérêt exceptionnel que présente pour l'histoire de la NouvelleFrance cette documentation abondante et variée que constituent les minutes notariales, étant bien entendu qu'il serait vain d'analyser le contenu des archives fiscales puisqu'elles sont inexistantes pour la période antérieure à la conquête anglaise. Bien que considérablement négligés jusqu'à présent, ces documents d'une rare qualité et quantité ne sont cependant pas passés totalement inaperçus au Québec. Déjà en 1921, P.-G. Roy, ce travailleur infatigable à qui tous les mérites n'ont pas été rendus, faisait ressortir auprès d'un public surtout préoccupé de "petite histoire" et de généalogie la valeur de l'inventaire après décès. ${ }^{3}$ Quelques années plus tard, dans le même but de venir en aide aux amateurs de généalogie, il inaugurait la collection des inventaires des contrats de mariage et des greffes des notaires. Plus près de nous, Fernand Ouellet, dans un volume déjà célèbre, ${ }^{4}$ Cameron Nish dans un essai original ${ }^{5}$ et R.-L. Séguin dans une synthèse ${ }^{6}$

3 P.-G. Roy, "L'ameublement d'un seigneur canadien sous l'Ancien Régime”, RAPQ (1921-1922) : 237-238.

4 Fernand Ouellet, Histoire économique et sociale du Québec 1760-1850 (Fides, Montréal et Paris, 1966), 639 p.

5 Cameron Nish, Les Bourgeois gentilhommes de la Nouvelle-France 1729-1748 (Fides, Montréal et Paris, 1968), 202 p.

6 R.-L. Séguin, La Civilisation traditionnelle de l" Habitant" aux 17e et 18e siècles (Fides, Montréal, 1973, 2e éd., revue), $701 \mathrm{p}$. 
dont la clarté n'est pas la qualité maîtresse, ont utilisé plus ou moins abondamment et avec un bonheur inégal les archives notariales ${ }^{7}$. Malgré cette attention particulière, encore timide il faut l'avouer, portée aux minutes notariales ces dernières années, cette documentation est toujours l'objet d'une indifférence étonnante et d'une utilisation par trop limitée ${ }^{8}$. Nous voulons donc simultanément plaider la cause de ces vieux papiers et, tout en mettant en relief les objectifs visés, proposer une méthode d'exploitation et d'utilisation pour deux catégories de documents en particulier: les contrats de mariage et les inventaires après décès.

\section{RESSOURCES, CRITIQUE ET EXPLOITATION DES ACTES NOTARIÉS}

Le contrat de mariage demeure le document notarié le plus abondant et le plus commode à dépouiller, grâce entre autres aux fiches-types mises au point par le Centre de Roland Mousnier ${ }^{9}$ qui en facilitent et en accélèrent considérablement la cueillette. C'est un acte stéréotypé, assez bref en général, qui se présente habituellement de la facon suivante. La première partie du document, l'intitulé, nous offre dans les meilleurs cas des renseignements substantiels sur les futurs époux, leurs parents et amis, témoins ou non à la signature du contrat, soit leurs noms et prénoms avec le pseudonyme quelquefois, leurs qualités

7 Jacques Mathieu, bien que très succinctement (RHAF, décembre 1969: 409-410) en a aussi souligné l'utilité pour une étude de la vie à Québec au milieu du XVIIe siècle. Un siècle plus tôt, Monseigneur Cyprien Tanguay les avait utilisées à quelques reprises pour compléter les indications fournies par les registres paroissiaux.

${ }^{8} \mathrm{Au}$ moment de publier cet article est paru le bel ouvrage de Louise Dechêne, Habitants et marchands de Montréal au XVIIe siècle (Plon, Montréal et Paris, 1974,588 p.) qui repose, en très grande partie, sur l'exploitation des minutes notariales et qui vient répondre à quelques-unes des questions posées dans ce texte, du moins en ce qui à trait au XVIIe siècle. C'est la première synthèse sur la Nouvelle-France qui utilise cette documentation complexe à des fins d'histoire économique et sociale. A cet égard, ce livre remarquable vient renouveler les méthodes d'investigation de l'histoire du régime français. Sorbonne.

${ }^{9}$ Le Centre de Recherches sur la civilisation de l'Europe moderne à la 
le cas échéant,10 leurs professions, leurs âges exceptionnellement ${ }^{11}$, leurs domiciles enfin. Puis viennent les diverses clauses qui fixent les conditions matérielles du mariage et qui sont la raison essentielle du contrat. On établit d'abord le régime de la coutume suivi par les futurs époux, en l'occurrence, et sauf exception, la coutume de Paris obligatoire au Canada depuis 1664 et qui stipule la communauté de biens pour les futurs mariés. Suit l'état des biens des conjoints, soit la dot apportée par la future et plus rarement l'estimation de la fortune de l'époux. ${ }^{12}$ Enfin on précise le douaire, le préciput, le droit de renonciation à la communauté pour la femme et les donations entre-vifs s'il y a lieu. L'acte se termine par la liste des signatures des contractants et des témoins présents à la rédaction du document.

Avant l'exploitation de cette documentation, une opération s'impose à toute enquête qui veut utiliser les contrats de mariage à des fins de description sociale: c'est de juger de la représentativité de la source elle-même. Quelle est la fréquence du recours au notaire par rapport au nombre de mariages célébrés et quel est le nombre des contrats conservés par rapport à la totalité des mariages conclus? Le seul moyen de le vérifier oblige à recourir aux registres paroissiaux de mariage, complément indispensable aux actes notariés qui permet d'établir la proportion des gens mariés qui fréquentent l'étude du notaire. Dans un second temps, il importe de mesurer l'écart, souvent fort négligeable d'ailleurs, entre la quantité d'actes rédigés et conservés jusqu'à nos jours. ${ }^{13}$ Les sondages effectués en France dans diverses régions ont permis d'établir les proportions suivantes pour le dix-huitième siècle: Paris: 75\% ${ }^{14}$; Bordeaux:

10 On aurait tort de négliger ces fameuses qualités ou "épithètes d'honneur" auxquelles les gens d'Ancien Régime tenaient à tout prix et qui sont à plus d'un titre révélatrices de leur rang social. Au Canada, dans une nouvelle géographie et conditionnées par un milieu social distinct de celui de la métropole, les qualités ont pu varier, être couramment usurpées, ou perdre leur véritable signification sociale. Cela reste à voir. Le juriste Loyseau, dans son Traité des ordres et simples dignitez, publié au début du dix-septième siècle, en a dressé une liste complète partiellement valable pour le dix-huitième. Du titre de "sieur" à celui de "chevalier" l'échelle des qualificatifs y est exhaustive.

11 Le plus souvent, le notaire se limite à la mention de la minorité ou de la majorité des contractants.

12 En effet, dans les contrats rédigés sous la Coutume de Paris, l'apport du mari est assez fréquemment passé sous silence.

13 Cela n'est pas toujours possible, mais chaque fois que le répertoire tenu par le notaire existe, le calcul en est aisé.

14 Adeline Daumard et François Furet, "Structures et relations sociales à Paris au XVIIIe siècle", Cahiers des Annales, no 18 (Paris, 1961): 9. 
65\% ${ }^{15}$; Dijon: $82 \%{ }^{16}$; Agen : $87 \%^{17}$; Toulouse : $90 \%{ }^{18}$; Lyon: $95 \%{ }^{19}$. Il y a tout lieu de croire que les contrats rédigés en Nouvelle-France, particulièrement au dix-huitième siècle, sont dans le même ordre de grandeur en ce qui a trait à la représentativité et à la conservation. ${ }^{20}$

Les contrats de mariage, en France du moins, demeurent donc assez bien représentatifs de la population en général, bien que le groupe le moins bien représenté (il va sans dire que les ecclésiastiques et les célibataires nous échappent totalement) soit celui des plus pauvres dont l'indigence et la précarité des conditions matérielles les empêchent d'avoir recours au notaire. Quoi qu'il en soit et en dépit de cette lacune, les contrats de mariage constituent à toutes fins pratiques avec les registres paroissiaux à peu près les seuls actes précis susceptibles de nous désigner les travailleurs manuels et les couches les plus humbles de la population. D'autre part, et c'est là un avantage considérable par rapport à d'autres catégories de documents, les contrats de mariage, à cause de leur abondance et de leur continuité, offrent la possibilité d'une histoire sérielle et d'une interprétation statistique et quantitative par l'ordinateur. C'est à travers eux que peuvent être saisis durant une longue période les grands traits d'une évolution séculaire.

Ces prémisses étant acceptées, que tirer du luxe de détails contenus dans ces archives? Evidemment, les contrats de mariage nous présentent d'abord des éléments d'information inté-

15 Etienne Dravasa, "Les classes sociales au XVIIIe siècle à Bordeaux d'après les contrats de mariage", Revue juridique et économique du Sudouest, XIV, 4 (1963) : 977.

16 Maurice Garden, "Niveaux de fortune à Dijon au milieu du $18 \mathrm{e}$ siècle", Cahiers d'Histoire, IX, 3 (1964) : 220.

17 Anne Marie Petit, "Mariages et contrats de mariage à Agen en 1785 et $1786 "$, Annales du Midi (1960) : 218.

$18 \mathrm{~J}$. Godechot et S. Moncassin, "Structures et relations sociales à Toulouse en 1749 et en 1785", Annales historiques de la Révolution française, XXXVII, 2 (1965) : 129.

19 Maurice Garden, "Le contrat de mariage lyonnais, une source de l'histoire sociale du XVIIIe siècle", Actes du $89 e$ Congrès national des sociétés savantes - Lyon 1964 - (Paris, 1965), 52.

20 Des recherches que nous menons depuis un an avec nos collègues Jean Blain et Louis Michel, bien secondés par une équipe d'étudiants laborieux, nous permettent de croire à l'exceptionnelle richesse quantitative de cette documentation. Notre but, partiellement réalisé à ce jour, est de mettre sur fiches tous les contrats de mariage rédigés dans le gouvernement de Montréal entre 1671 et 1781. En effet, l'avantage de travailler avec une population réduite ne nous oblige pas à un échantillonnage, mais nous permet plutôt d'espérer, grâce aux subventions du gouvernement du Québec, que tous les documents de mariages tenus par les notaires seront dépouillés. 
ressants pour calculer les taux d'alphabétisation en NouvelleFrance dans la mesure où les seules signatures qui apparaissent au bas de l'acte peuvent servir d'indices pour estimer la proportion des analphabètes. Une telle série d'actes nous permettra sans doute de constater une variation (une dégradation peutêtre?) de l'alphabétisation dans la colonie entre le dix-septième siècle, le début et le milieu du dix-huitième siècle, soit entre les immigrants nés en France et les premières générations de Canadiens nés et instruits au pays. ${ }^{21}$

D'autre part, et c'est là son intérêt exceptionnel, cette documentation très riche est essentielle pour qui veut étudier la composition socio-professionnelle d'une population et sa topographie sociale. Tout d'abord, les indications de professions données au contrat nous renseignent utilement sur la structuration professionnelle de la colonie. Bien plus, les comparaisons de père à fils en ce qui a trait au métier pratiqué et au domicile indiqué autorisent l'historien à analyser la mobilité ou la pérennité professionnelle et géographique. L'ascension sociale et les mouvements de population en Nouvelle-France deviennent dès lors des entités quantifiables.

Enfin, l'institution du mariage, puisqu'elle donne lieu généralement à la rédaction d'un contrat, nous fournit toutes les composantes nécessaires pour bien délimiter et cerner ce qu'un historien français appelait récemment le "groupe d'existence", donnée capitale qui constitue l'une des notions premières d'une histoire à caractère social. En effet, le groupe par excellence ne serait-il pas celui

... composé de gens qui exercent des professions diverses 22 ... mais qui sont unis par plusieurs caractères: un idéal social commun; une estime sociale, qui est à double aspect: intérieure au groupe, la conscience que le groupe a de luimême, de son rôle, de son importance, de ses particularités dans la société d'ensemble; estime extérieure au groupe: l'image que la société d'ensemble ou d'autres groupes sociaux se font du groupe considéré et le jugement de valeur qu'ils portent sur lui; autres caractères: des activités de même nature,

21 Une fois ces taux établis pour la Nouvelle-France, la comparaison sera aisée avec ceux de la France puisqu'ils ont été compilés par Maggiolo à la fin du XIXe siècle. Voir à ce sujet l'article de M. Fleury et P. Valmary, "Les progrès de l'instruction élémentaire de Louis XIV à Napoléon III d'après l'enquête de Louis Maggiolo (1877-1879)", Population (1957).

22 Exceptionnellement le groupe d'existence va coïncider avec le groupe professionnel. En France, l'un des exemples les mieux connus est celui des vignerons dont la tendance à l'endogamie professionnelle a été mise en évidence par Marcel Couturier dans: Recherches sur les structures sociales de Châteaudun 1525-1789 (Paris, S.E.V.P.E.N., 1969). 
des ressources de nature semblable, ce qui est plus important peut-être que leur niveau; des genres de vie semblables ... une psychologie collective... des intérêts communs, des opinions et idées voisines. ${ }^{23}$

Une des marques extérieures de ce groupe qui permet de le bien délimiter, c'est le mariage de ses membres entre eux et ainsi, l'intermariage, constante sociale à laquelle à peu près toutes les sociétés ont obéi dans la majorité des unions, devient l'instrument essentiel pour servir à la définition et à la délimitation des groupes sociaux. En définitive, si l'on admet qu'un des caractères essentiels et permanents du groupe social c'est cet intermariage de ses membres, on peut à la limite avancer que le groupe social réel est constitué des gens qui se marient entre eux. Qui épouse qui? C'est en comparant la profession et qualité $\mathrm{du}$ futur époux ${ }^{24}$ avec celles de son beau-père qu'il devient possible d'isoler sur un grand nombre de cas des noyaux d'alliances composés de groupes de professions et qualités semblables ou diverses et autour desquels vont se former des halos plus ou moins flous allant en décroissant. Ces unions à base de professions et qualités offrent tous les éléments constitutifs pour une appréciation globale du groupe d'existence. Ainsi, par exemple, en isolant tous les futurs maris qui portent la profession de marchand, ${ }^{25}$ il s'avère possible de reconstituer le groupe social dont ils font partie en confrontant leur état professionnel avec celui du père de la future. Dans cette optique, nous pourrons découvrir les tendances plus ou moins prononcées de ce groupe à l'endogamie professionnelle et les diverses professions et qualités, ${ }^{26}$ le cas échéant, qui donnent lieu à des

23 Roland Mousnier, "Problèmes de méthode dans l'étude des structures sociales des XVIe, XVIIe, XVIIIe siècles", La Plume, la Faucille et le Marteau (Paris, P.U.F., Collection Hier, 1970), 14.

24 Dans une société patrilinéaire, il faut partir du futur époux et rapprocher ses données sociales de celles de son beau-père. Il est bien entendu qu'en cas de viduité de la future, c'est la profession de son premier mari qui sera retenue pour la comparaison.

${ }_{25}$ Le seul moyen véritable de définir ces groupes d'alliances, c'est de se baser sur le futur époux et de retenir comme critère de comparaison sa profession et qualité. On peut ainsi regrouper tous ceux qui exercent une profession similaire (marchands de fourrures par exemple) ou qui participent à une même forme générale d'activité (la "marchandise" ou l'artisanat) et rechercher les unions qu'ils contractent.

26 Dans la métropole, les épithètes d'honneur couramment attribuées aux marchands sont celles de "sieur" et de "bourgeois". La plupart du temps, elles ont une signification qui témoigne du rang social et de l'état de fortune. Il est habituellement normal qu'un futur époux qualifié s'allie à un beau-père également pourvu d'une épithète honorifique. Ces unions de qualités semblables sont évidemment des données importantes pour la 
unions. ${ }^{27}$ Dans le même sens et avec un très grand nombre d'exemples s'offre la possibilité d'étayer la thèse de Cameron Nish qui a réussi à démontrer partiellement l'homogénéité des élites en Nouvelle-France. En découvrant ainsi les principaux réseaux d'alliances et les cercles d'unions les plus importants, ${ }^{28}$ on pourra préciser la structure sociale de la Nouvelle-France, entendez l'analyse des groupes et de leurs rapports, et identifier les groupes d'existence les plus apparents.

Evidemment, tous les individus n'obéissent pas à l'intermariage et des exceptions existent puisque les documents notariés nous révèlent des mésalliances et des ascensions sociales par le mariage qui sont le fait davantage des veufs et des veuves, enclins souvent à sacrifier leur statut social pour se trouver un partenaire qui accepte d'élever les enfants d'un premier lit. ${ }^{29}$ Mais si nous opérons sur un grand nombre de cas, ces exceptions ne limitent pas la valeur des résultats et s'estompent d'ellesmêmes. Quoi qu'il en soit, diverses indications au contrat nous permettent de discerner ces cas aberrants. La plupart du temps une clause de séparation de biens (la communauté est généralisée sous le régime de la Coutume de Paris), une différence substantielle entre les deux apports, quand ils sont indiqués, ou une donation unilatérale témoignent d'une différenciation sociale.

Une fois les groupes sociaux discernés et assez bien délimités, il importe de rechercher le niveau de fortune de ses membres, ses ressources et ses conditions de vie matérielle. A cet effet, des éléments plus ou moins satisfaisants contenus dans le contrat peuvent nous renseigner sommairement sur ces ques-

définition du groupe social. En Nouvelle-France, il reste à voir si elles ont la même signification et, si oui, à établir la proportion des alliances qui respecte la réciprocité des titres honorifiques.

27 Pour une illustration de cette méthode, consulter le bel ouvrage de Yves Durand intitulé: Les fermiers généraux au XVIIIe siècle (Paris, P.U.F., 1971).

28 Dans cette tâche, on peut s'aider des listes de témoins parents et amis qui assistent à la signature du contrat. Quand il est bien rédigé, on y retrouve les professions, qualités et domiciles des témoins, indications précieuses pour reconstituer les parentèles, les attaches et les relations sociales du groupe.

29 Pour obvier à ces mésalliances et pour empêcher que les biens des héritiers soient dilapidés, la législation d'Ancien Régime avait défendu aux veufs et aux veuves, par l'Edit des Secondes Noces de 1560, de donner à leur seconde épouse ou époux plus d'une part d'enfant au moment de contracter l'union. 
tions. Assurément, le chiffre de la dot et de l'apport du futur ${ }^{30}$ totalisé apparaît comme une source indicatrice du niveau de fortune des contractants. Mais il faut bien se garder de prendre pour acquit que les résultats obtenus donnent la mesure exacte des ressources du jeune ménage et du groupe social qu'il représente. En effet, pour une même catégorie sociale les dots de plusieurs futures épouses peuvent varier considérablement suivant le nombre de filles à marier à l'intérieur de la même famille. Si l'on n'y prend garde, il est aisé de fausser complètement l'échelle des fortunes à l'entrée en ménage et placer un ou des couples à un niveau inférieur qui n'est pas réellement le leur. A cet égard, une étude complète des familles, sur laquelle nous reviendrons, s'avère indispensable. Dans le même sens, il faut s'assurer que la dot de la future représente bien toute sa part d'héritage et non pas simplement un "avancement d'hoirie".

D'autre part, la dot considérée globalement dans sa totalité est peut-être moins révélatrice que la portion de cet apport qui doit entrer en communauté, le plus souvent le tiers ou la moitié de la somme. Car la fraction qui doit rester propre à la femme et revenir à son côté et ligne (habituellement les immeubles) ne peut être aliénée par le mari dont le seul droit réside dans l'utilisation du revenu de ses propres. ${ }^{31}$ Evidemment, de deux dots égales en chiffre, celle dont $75 \%$ des biens restent propres à la future est nettement inférieure en possibilités d'investissements pour le mari à celle dont le quart demeure propre à la femme. Au surplus, la nature des composants est à analyser au même titre que leur niveau et leur proportion s'il est vrai qu'une dot composée essentiellement d'argent liquide (les deniers comptants) et d'immeubles (terres ou maisons) est à interpréter différemment de celle qui ne contient que des "meubles meublans" et quelques écus.

30 Il n'est pas inutile de rappeler que dans les contrats de mariage rédigés en vertu de la Coutume de Paris les renseignements sur les biens présents du futur conjoint font souvent défaut. D'autre part, les premiers dépouillements effectués nous ont permis de constater le peu de renseignements fournis par les futurs quant aux biens possédés. Dans de nombreux cas, le notaire se contente d'indiquer laconiquement que les futurs époux se prennent avec leurs biens et droits, témoignage sans doute de leur faible valeur.

31 A moins que le conjoint soit autorisé par sa femme à vendre le bien propre. Même en ce cas, il se voit dans l'obligation de remployer le capital en biens qui deviendront propres à la future et à ses héritiers. Ces distinctions sont surtout valables pour les groupes supérieurs dont les membres, possesseurs de biens importants, peuvent faire valoir la notion de propres. 
Enfin, on peut faire observer que les clauses accessoires comme le douaire et le préciput contenus dans le document ne sont pas d'une grande utilité pour établir la fortune du couple. Ces deux éléments à partir desquels il serait bien aléatoire de vouloir connaître les ressources du mari offrent cependant la possibilité d'instituer des ordres de grandeur valables pour chacune des catégories représentées et de les comparer entre eux.

En dépit des quelques précisions qu'ils nous fournissent sur les conditions matérielles du futur ménage, il faut bien reconnaître avec Jean-Yves Tirat ${ }^{32}$ qu'il est particulièrement ardu de définir au moyen des contrats de mariage les niveaux de fortune réels des futurs ménages. Selon lui, la dot ou les apports combinés donnent assez rarement la mesure exacte ou même rapprochée (nous n'irions pas si loin) du niveau de richesse ou de pauvreté des conjoints ou de leurs parents. "La dot peut varier du simple au quadruple, suivant le nombre d'enfants et surtout de filles à marier. Il faudrait donc supposer que la dimension des familles ait été la même dans toutes les couches de la société pour que le calcul statistique y trouve son compte." 33 Toutefois, même en admettant que le contrat de mariage soit insuffisant pour isoler l'état des fortunes, il demeure cependant notre seule ressource pour tenter d'approcher les niveaux de fortune réels des gens en âge de se marier. $\mathrm{Au}$ demeurant, la dot, si elle ne permet pas d'obtenir une connaissance des niveaux de fortune individuels, nous renseigne par comparaison sur les disponibilités des différentes catégories sociales.

D'autre part, bien qu'il soit entaché de ces quelques défauts, ce contrat notarié, par ses indications sur les dots, les douaires et les préciputs, devient en dernier ressort une admirable source d'histoire sérielle. A cet égard, sur une longue période et à l'intérieur d'une même catégorie sociale, l'augmentation ou la diminution constante du chiffre des dots, des douaires et des préciputs devient révélatrice d'une période de prospérité ou de récession économique et, au même titre qu'une série de prix, ces trois variables viennent témoigner des pulsations de la vie économique.

Si le contrat de mariage est indispensable à une étude sociale, il n'est cependant pas dissociable d'un autre type de

32 Jean-Yves Tirat, "Problèmes de méthode en histoire sociale", Revue d'histoire moderne et contemporaine, X, 3 (1963) : 211-218.

33 Ibid.: 214. 
document notarié: l'inventaire après décès. Cet acte complexe est le complément nécessaire au précédent dans la mesure où il nous permet de préciser certaines dénominations professionnelles trop vagues dans les contrats de mariage. Dans de nombreux cas, la profession indiquée à l'acte de mariage nous laisse perplexe et ne nous offre pas la possibilité d'atteindre l'activité économique réellement exercée par certains groupes, ce qui interdit tout classement sérieux à l'intérieur de catégories socioprofessionnelles précises. Ainsi, qui se cache derrière le titre de marchand-bourgeois de Montréal? L'inventaire après décès par les stocks de marchandises qu'il nous décrit et les listes d'outils qu'il détaille sera souvent susceptible de nous révéler la signification exacte de ces vagues appellations. D'autre part, il faut remarquer que le contrat de mariage n'est apte qu'à nous fournir une structure sociale à l'entrée en ménage (une population relativement jeune donc) et ne nous permet d'isoler qu'un instant de la vie, bien important toutefois, la situation au moment du mariage. Pour connaître l'évolution d'un couple ou d'un groupe social, il faut reconstituer les carrières et les vies et atteindre la fortune à son apogée. L'inventaire de la succession est tout à fait approprié à cette recherche.

Cet acte notarié plus difficile à exploiter, dont PierreGeorges Roy, en 1921, révélait chez nous l'utilité pour l'étude de la vie familiale de nos ancêtres, se présente habituellement sous la forme suivante. Plus volumineux en général que l'acte de mariage, (il contient souvent quelque dizaines de feuilles alors que le précédent se résume la plupart du temps à trois ou quatre) il donne d'abord la liste des membres de la famille intéressés à la succession comme créanciers ou héritiers. Puis vient une description que l'on peut tenir pour fidèle, pièce par pièce, des "meubles meublans" (mobilier, tableaux, livres) et des deniers comptants, s'ils existent. Chez les commerçants seront prisées les marchandises et chez les ruraux, les bâtiments de ferme, les instruments aratoires, le bétail, "les bleds" engrangés et ceux en voie d'être moissonnés. Enfin, et ce n'est pas là son moindre intérêt, on y retrouve, quand il est bien dressé, une liste relativement complète des papiers qui concernent la succession du défunt. Mais alors que le contrat de mariage atteint dans les meilleurs cas des taux de représentativité exceptionnelle, l'inventaire, quant à lui, est plus riche par son contenu que par la proportion des gens qu'il nous permet d'atteindre. 
Les sondages effectués en France ${ }^{34}$ n'ont jamais obtenu des pourcentages supérieurs à $20 \%$ dans le rapport décès comptabilisés-inventaires établis, ce qui amoindrit la valeur de cette documentation. Il faut bien le dire, l'inventaire après décès est un acte plutôt exceptionnel et relativement rare qui nous offre une image assez sélective de la société. Nécessité par l'existence d'une tutelle d'enfants mineurs, provoqué par un différend entre les héritiers, indispensable à la dissolution d'une communauté de biens, ce document notarié, qui peut coûter assez cher par les nombreuses vacations d'huissier qu'il requiert, n'est pas à la portée de chacun d'autant plus qu'il faut un minimum de biens pour qu'un inventaire soit dressé. Dans ces conditions, beaucoup de successions qui ne font pas problème et la plupart de celles qui ont trait aux couches inférieures de la population ne sont pas closes par des inventaires. En définitive, ils ne nous offrent que des cas individuels à partir desquels il serait hasardeux de généraliser. A cet égard et compte tenu de ces lacunes, “ . . ills ne doivent être employés que comme complément d'une étude plus vaste, pour préciser d'autres données générales fournies par des documents plus largement ouverts à toutes les catégories sociales et professionnelles" ${ }^{35}$.

Quoi qu'il en soit, la diversité de l'information qu'il nous livre et la richesse de son contenu en ont fait un document archivistique de tout premier ordre retenu d'emblée par les historiens tournés vers le social, l'économique et le culturel. Les renseignements qu'il nous communique sont de nature diverse. La fortune mobilière d'une communauté est souvent bien détaillée dans les premières feuilles et le compte en est vite fait, évidemment en y ajoutant la crue le cas échéant. ${ }^{36}$ D'autre part, l'étude de certains objets caractéristiques (mobilier de cuisine, literie, argenterie, tableaux, images, livres ...) nous procure des indications sur le style de vie, le décor de l'habitation, ${ }^{37}$ la forme de piété pratiquée et le genre de culture recherchée. La fortune

${ }^{34}$ Il s'agit pour établir le taux de représentativité de la source de comparer par le dépouillement des registres de sépulture le nombre de décès annuels et la quantité d'inventaires rédigés.

35 Maurice Garden, "Les Inventaires après décès: Source globale de l'histoire sociale lyonnaise ou juxtaposition de monographies familiales?", Cahiers d'Histoire, XII (1967) : 159.

36 Quand l'estimation n'est pas faite à sa juste valeur, il faut y ajouter la crue ou le parisis qui, dans la coutume de Paris, est une augmentation de $25 \%$ par rapport à l'évaluation du commissaire-priseur.

37 L'examen de l'ameublement intérieur intéresse bien sûr l'histoire sociale. On peut penser qu'il y a comme un élément de la définition du groupe social dans le décor de la vie. 
immobilière, son histoire, son évolution et ses différentes étapes peuvent être reconstituées grâce aux papiers énumérés en fin d'inventaire qui nous renvoient généralement à d'autres actes notariés. Les références au contrat de mariage, aux contrats d'achats ou de ventes de maisons, de terres, de commerces, sont de toute première utilité pour la reconstitution de l'histoire de la fortune. La liste des dettes actives ou passives est à rechercher minutieusement pour ce qu'elle nous instruit sur les formes de pouvoir économique et les zones géographiques à l'intérieur desquelles il se fait valoir. ${ }^{38}$

Les renseignements fournis pour l'étude du social proprement dit sont peut-être plus importants encore et c'est là assurément une contribution essentielle de cet acte notarié. En effet, il est temps de remettre à l'honneur des disciplines longtemps méprisées, la généalogie et l'histoire des familles, et sans l'inventaire après décès, la reconstitution des familles et l'étude de la mobilité sociale demeurent impossibles. En nous procurant les références à des contrats de mariage et à des inventaires antérieurs il devient une source primordiale pour la généalogie considérée désormais d'un point de vue social. ${ }^{39}$

\section{CONCLUSION}

Les contrats de mariage et les inventaires après décès nous sont apparus les deux documents notariés privilégiés pour une histoire sociale de la Nouvelle-France. Leur consultation n'est cependant pas exclusive et leur emploi n'interdit pas de recourir à des actes complémentaires du même genre ou non. Ainsi, les précieux registres paroissiaux, dont nous possédons une collection admirable et que Hubert Charbonneau et son équipe sont à exploiter à la suite de Monseigneur Tanguay, constituent une source de première valeur dont l'utilisation sur une longue période est indispensable à toute étude qui se veut sociale. ${ }^{40}$

38 Ces différentes énumérations de dettes actives analysées chez les marchands de Montréal, par exemple, seraient susceptibles de nous révéler l'existence d'une zone économique périphérique dépendante de cette ville. Par la même occasion, l'étude des relations ville-campagne en sortirait enrichie.

39 Consulter à ce propos le livre de Roland Mousnier qui a pour titre: Le Conseil du Roi de Louis XII à la Révolution (Paris, P.U.F., 1970).

40 Marcel Trudel, il y a quelques années, dans un article intitulé: "Les débuts d'une société: Montréal, 1642-1663. Etudes de certains comportements sociaux" (RHAF, septembre 1969), à partir d'un échantillon assez réduit (85 mariages), avait tenté de saisir par le biais des registres paroissiaux les lignes de force de certaines relations sociales au début de la société montréalaise. 
Ils nous permettent de retrouver les quelques individus qui n'ont pas fait rédiger de contrat de mariage. Leur utilité est évidente quand il s'agit de confronter les données qui apparaissent dans l'acte notarié (professions, qualités, signatures), ce qui permet une critique nécessaire de la documentation notariale. Ils offrent, au même titre que les minutes de notaires, l'avantage de saisir sur une période étendue cette structure sociale dont une des caractéristiques essentielles est la longue durée. Enfin, des archives notariales accessoires et complémentaires pourront éventuellement combler le vide temporel qui s'étend entre le mariage et le décès. Ainsi, les contrats de concessions et de ventes (terres, maisons...), les baux de location, les comptes de tutelles, les partages de successions ne sont certes pas négligeables. En dernier ressort, il ne faudrait pas passer sous silence les innombrables contrats d'apprentissage révélateurs des conditions de l'artisanat, de la mobilité sociale et géographique dans la colonie, ${ }^{41}$ et les centaines de testaments qui dorment dans les liasses et dont l'analyse minutieuse nous permettrait d'approcher la pratique religieuse et les comportements des Canadiens devant la mort. ${ }^{42}$

Après avoir dépouillé et mis sur fiches tous ces documents délaissés et dont la lecture n'est pas toujours facile, leur exploitation, facilitée par la mécanographie, rendra compte des réalités sociales en Nouvelle-France. Discerner les groupes sociaux, établir les échelles de fortune, reconstituer l'histoire sociale des familles, classifier et hiérarchiser les statuts, voilà un programme de travail étoffé et de longue haleine mais combien riche en promesses.

41 Déjà, dans Economie et Société en Nouvelle-France (Québec, P.U.L., 1960), Jean Hamelin, attentif aux avantages de cette documentation, invitait les historiens à y rechercher les éléments pour une étude de la condition ouvrière. Peter N. Moogk s'est intéressé à ce problème en dépouillant quelques centaines de contrats d'apprentissage. "Apprenticeship: indentures: A key to artisan life in New France", The Canadian Historical Association, Historical Papers (1971) : 65-83.

42 Récemment, un historien français, Michel Vovelle, utilisant quelques milliers de testaments provençaux, a pu avec beaucoup de justesse analyser les attitudes collectives devant la mort au XVIIIe siècle et mesurer le taux de déchristianisation dans la France du sud. Piété baroque et déchristianisation en Provence au dix-huitième siècle (Paris, Plon, 1973), $697 \mathrm{p}$. 


\section{A N N EX ES}

I

\section{EXEMPLE DE CONTRAT DE MARIAGE ${ }^{43}$}

Par devant les notaires royaux fut présent Sieur Jacques Hervieux négociant demeurant en cette ville natif de la paroisse Notre-Dame de Montréal fils de sieur Léonnard Jean Hervieux marchand bourgeois de cette ville y demeurant et de feüe dame Catherine Magnant son épouse pour luy et en son nom d'une part et sieur Jacques Quenel Fonblanche aussi marchand bourgeois de cettedite ville cy devant veuf de deffunte damoiselle Marie Anne Thuillier Lacombe sa première femme stipulant en cette partie pour damoiselle Marie Jeanne Quenel sa fille à ce présente et de son consentement d'autre part. Lesquels parties de l'agréement et consentement de leurs parents et amis cy après nommés scavoir de la part dudit sieur Jacques Hervieux futur époux, ledit sieur Hervieux son père, sieur Jean Baptiste Lecomte Dupré et damoiselle Marie Anne Hervieux son épouse, sieur Jean Baptiste Hervieux et damoiselle Charlotte de Lamargue son épouse, sieur Toussaint Pothier et damoiselle Geneviève Hervieux son épouse tous marchands de cette ville, frère Antoine Hervieux Récollet, damoiselle Michelle Hervieux et sieur Louis François Hervieux et damoiselle Louise Quenel son épouse, Luc de la Corne de Chap escuier sieur de Saint-Luc officier des troupes de la marine et dame Marie Anne Hervieux son épouse ses frères et sœurs, damoiselle Louise Lecompte veuve de feu le sieur Antoine Magnan Lespérance vivant marchand bourgeois de cettedite ville, damoiselle Marie Geneviève Hervieux épouse de sieur Guillaume Séguin sa tante, damoiselle Louise Lecomte Dupré sa nièce, damoiselle Louise Marie Anne et Marguerite Magnan Lespérance et Louise Gignière ses cousines, messire Poulin prêtre et curé de la paroisse de l'isle Jésus.

Et de la part de ladite damoiselle Jeanne Quenel dudit sieur Quenel son père et damoiselle Marie Franquelin sa belle-mère, damoiselle Catherine et Marie Anne Quenel ses sœurs, Jean Quenel, sieur Jean Baptiste Labrêche, messire Joseph Lacombe prêtre et curé de Lachesnaye sieur pierre Guy et damoiselle Jeanne Lacombe son épouse, Jacques Moquin et Marie Joseph Lacombe, Jeanne Prudhomme veuve de feu sieur Dominique Thaumur Lacource, Jeanne (?) veuve de feu le sieur Lafatigue ses oncles et tantes, sieur Pierre LePette Lahaye et Marie Joseph Cauchois son épouse et sieur Louis Prudhomme et Louis de Lamargue Marin son épouse ses cousins et cousines et sieur Pierre Couraud Lacoste marchand de cette diteville.

Ont fait ensemble les accords et conventions de mariage qui suivent c'est à scavoir que ledit sieur Jacques Hervieux et ladite damoiselle Jeanne

43 Nous n'avons pas reproduit le texte d'un inventaire après décès. Il est facile d'en consulter en dépouillant les Rapports de l'Archiviste de la province de Québec. Cameron Nish, il y a quelques années, avait publié dans Actualité économique, celui de François Etienne Cugnet. Le contrat de mariage que nous reproduisons ici est tiré de l'étude de Jean-Baptiste Adhémar déposée aux Archives Nationales du Québec à Montréal. 
Quenel se sont promis et promettent de prendre pour mary et femme par nom et loy de mariage, pour y celluy faire et solemniser en face de notre mère sainte Eglise catholique, apostolique et romaine le plus tost que faire se pourra et qu'il sera avisé et délibéré entre eux leurs parents et amis si Dieu et notre mère sainte Eglise y accordent consentement.

Seront lesdits sieur et damoiselle futurs époux unis et communs en tous biens meubles acquets et conquets immeubles qu'ils auront et feront pendant et constant leur futur mariage suivant et au désir de la coutume de Paris suivi en ce païs et à laquelle ils se soumettent comme aussi les propres desdits futurs époux entreront dans ladite future communauté tant ceux qui leur sont escheus que ceux qui leur échéront tant par successions, donnations qu'autrement à l'esfect duquel dernier article ils ont du consentement de leursdits parents et amix cy dessus nommés dérogé à toutes lois et coutumes à ce contraire auquels ils ont expressement rennoncé et rennoncent expressement par ces présentes.

$\mathrm{Ne}$ seront tenus aux dettes l'un de l'autre faites et crées avant leurs épousailles mais si aucunes y seront payé et acquittés par celluy d'eux qui les aura faites et sur son bien sans que l'autre en soit tenue en quelque manière que ce soit.

Ledit sieur futur époux a doué et doue ladite damoiselle future épouse du douaire coutumier ou de la somme de deux mille cinq cents livres de douaire préfix une fois payé à son choix et des enfants à naître dudit futur mariage. Duquel douaire préfix ou coutumier tel qu'il sera choisy jouir et avoir délivrance dès que douaire aura lieu sans estre tenue de le demander en justice pour seureté duquel douaire préfix ladite damoiselle future épouse et ses enfans auront leur hipotèque de ce jour sur tous les biens présens et avenir dudit sieur futur époux.

Le survivant desdits sieur et damoiselle futurs époux aura et prendra pour son préciput et hors part des biens de leur communauté scavoir ledit futur époux ses habits, linges, hardes, armes et bagage et ladite damoiselle future épouse ses habits, bagues, joyaux, linges, sa toilette, son lit et sa chambre garnie et en outre des meubles de ladite communauté tel que ledit survivant voudra choisir suivant la prisée de l'inventaire qui en sera faite et sans crue jusqu'à la somme de mille livres ou ladite somme en deniers comptans au choix dudit survivant.

$\mathrm{Au}$ surplus lesdits sieur et damoiselle futurs époux se prennent avec leurs biens et droits tous ceux qui leur seront écheus ou ceux qui leur écherront soit par successions, donnations ou autrement lesquels entreront en ladite future communauté comme il est dit cy dessus.

Sera loisible à ladite demoiselle future épouse et aux enfans à naître dudit futur mariage d'accepter ladite communauté ou y renoncer et en cas de renonciation pourront reprendre tout ce que ladite demoiselle future épouse aura apporté et luy sera advenu et escheu par succession, donnation ou autrement avec ses douaire et préciput tel que dessus sans être tenue des dettes ni 
hipotèques de ladite communauté. En cas qu'elle y eut parlé si fut obligé ou y eut été condamné dont elle et les siens seront acquittés et indemnisés par et sur les biens dudit futur époux. Et pour laquelle indemnité auront leur hipotèque sur tous les biens présens et avenir dudit futur époux.

Déclare ledit futur époux qu'il a à luy appartenant la somme de deux mille livres qu'il a gagné par ses peines et soins. Et en faveur et considération dudit futur mariage ledit sieur Hervieux donne audit futur époux son fils ce acceptant avec ladite damoiselle future épouse en avancement d'hoirie en sa succession future et en celle escheu de ladite deffunte Catherine Magnan mère dudit futur époux la somme de dix mille livres laquelle avec la susdite de deux mille livres fait ensemble celle de douze mille livres entrera en la future communauté comme acquise pendant ycelle.

Et pour la bonne et réciproque amitié que lesdits sieur et damoiselle futurs époux se portent l'un et l'autre ils se sont fait et font par ces présentes donnation viagère mutuelle égale et réciproque au survivant d'eux ce acceptant de tous et uns chacuns des biens meubles et immeubles présens et avenir sans aucune en excepter ny reserver tant des propres que d'acquets et conquets qui se trouveront appartenir au premier mourant au jour et heure de son décès à quelque somme qu'ils se puissent monter et valloir de quelque valeur qu'ils soient et en quelque lieux qu'ils se trouvent situés pour de tous lesdits biens en jouir par ledit survivant sa vie durante seullement pourvu qu'au jour et heure du décès dudit premier mourant il n'y ait aucuns enfans nés en légitime mariage auquel cas d'enfant ladite donnation sera nule et comme non faite et après leur décès les biens qui se trouveront pour lors seront partagés entre les héritiers desdits sieur et damoiselle futurs époux par moitié, et sans qu'ils soient tenus de donner caution pour la jouissance sinon à leur caution juratoire et pour faire insinuer ces présentes car ainsi promettant, obligeant rennoncant, fait et passé audit Montréal en la maison dudit sieur Fonblanche l'an mil sept cent quarante cinq le treize novembre après midy et ont lesdits futurs époux et parens et amis susnommés signés avec lesdits notaires lecture faite suivant l'ordonnance.

(Suit la liste des signatures)

\section{BIBLIOGRAPHIE POUR SERVIR À LA MÉTHODOLOGIE ET À L'UTILISATION DES ARCHIVES NOTARIALES}

Couturier, Marcel. Recherches sur les structures sociales de Châteaudun 1525-1789 (Paris, S.E.V.P.E.N., 1969), 294 p.

Daumard, Adeline. "Structures sociales et classement socio-professionnel: l'apport des archives notariales au $18 \mathrm{e}$ et au $19 \mathrm{e}$ siècle", Revue historique, CCXXVII, 1 (janvier-mars 1962) : 139-154. 
Daumard, Adeline et Furet, François.

Idem.

Dravasa, Etienne.

Durand, Yves.

Febvre, Lucien.

Garden, Maurice.

Idem.

Idem.

Idem.

Godechot, J. et Moncassin, S.

Goubert, Pierre.

Jurgens, Madeleine et Couperie, Pierre.
"Méthodes de l'histoire sociale. Les archives notariales et la mécanographie", Annales. Economies, Sociétés, Civilisation, XIV, 4 (octobre-décembre 1959) : 676-693.

Structures et relations sociales à Paris au XVIIIe siècle, Cahier des Annales no 18 (Paris, Armand Colin, 1961), 97 p.

"Les classes sociales au XVIIIe siècle à Bordeaux d'après les contrats de mariage", Revue juridique et économique du Sud-ouest, XIV, 4 (1963): 961-1012.

Les fermiers généraux au XVIIIe siècle (Paris, P.U.F., 1971), 664 p.

"Ce qu'on peut trouver dans une série d'inventaires mobiliers. De la Renaissance à la Contre-Réforme: changements de climat", Annales d'histoire sociale (janvier-juin 1941): 41-54.

"Le contrat de mariage lyonnais. Une source de l'histoire sociale du XVIIIe siècle", dans Actes $d u$ $89 e$ Congrès national des Sociétés Savantes (Lyon, 1964), Section d'Histoire moderne et contemporaine, t. II, vol. 1 (Paris, Imprimerie nationale, 1965): 51-75.

"Niveaux de fortune à Dijon au milieu du $18 \mathrm{e}$ siècle", Cahiers d'Histoire, IX, 3 (juillet-septembre 1964): 217-260.

Lyon et les Lyonnais au XVIIIe siècle (Paris, Les Belles-Lettres, 1970), $772 \mathrm{p}$. Plus particulièrement le chapitre intitulé: "Richesse, variété, ressources et limites des sources de l'histoire sociale de Lyon", 211-229.

"Les inventaires après décès: Source globale de l'histoire sociale lyonnaise ou juxtaposition de monographies familiales?", Cahiers d'Histoire, XII (1967): 153-173.

"Structures et relations sociales à Toulouse en 1749 et en 1785", Annales historiques de la Révolution française, XXXVII, 2 (avril-juin 1965): 129-169.

"Intérêt et utilisation historique des papiers de succession. Inventaires après décès, partages, comptes de tutelles", Revue d'histoire moderne et contemporaine, I, 1 (janvier-mars 1954) : 22-24.

"Le logement à Paris aux XVIe et XVIIe siècles: une source, les inventaires après décès", Annales. Economies, Sociétés, Civilisations, XVII, 3 (maijuin 1962): 488-506. 
Labrousse, Ernest. Voies nouvelles vers une histoire de la bourgeoisie occidentale aux XVIIIe et XIXe siècles (1700-1850), $\mathrm{X}$ Congrès international des Sciences historiques (Rome, 1955), Relazioni, vol. IV, Storia Moderna: 365-396.

Lafon, Jacques. Régimes matrimoniaux et mutations sociales. Les époux bordelais 1450-1550 (Paris, S.E.V.P.E.N., 1972), $345 \mathrm{p}$.

Lelièvre, Jacques. La pratique des contrats de mariage chez les notaires au Châtelet de Paris de 1769 à 1804 (Paris, Cujas, 1959), $402 \mathrm{p}$.

Mousnier, Roland. "Problèmes de méthode dans l'étude des structures sociales des XVIe, XVIIe, XVIIIe siècles", La Plume, la faucille et le marteau (Paris, P.U.F., 1970), 12-26.

Idem.

Le Conseil du Roi de Louis XII à la Révolution (Paris, P.U.F., 1970), 378 p.

Petit, Anne-Marie. "Mariages et contrats de mariage à Agen en 1785 et en 1786", Annales du Midi (1960): 215-229.

Poisson, Jean-Paul. "De quelques nouvelles utilisations des sources notariales en histoire économique (XVIIe - XXe siècle)", Revue historique, CCXLIX, 1 (janvier-mars 1973)': 5-22.

Poussou, Jean-Pierre. "Expérience aquitaine et méthodologie des contrats de mariage au XVIIIe siècle", Annales $d u$ Midi (1964): 61-76.

Tirat, Jean-Yves. $\quad$ "Problèmes de méthode en histoire sociale", Revue d'histoire moderne et contemporaine, X, 3 (juilletseptembre 1963): 211-218.

Vincent, Jacqueline. "Richesses et lacunes des actes notariés pour la connaissance des anciennes structures sociales: les contrats de mariage: Cannes de 1785-1815", Revue historique, CCXLI, 4 (octobre-décembre 1973): 363-402. 